\title{
Hidradenitis Suppurativa and Concurrent Psoriasis: Comparison of Epidemiology, Comorbidity Profiles, and Risk Factors
}

\author{
Andreas Pinter (D) - Georgios Kokolakis · Juergen Rech • \\ Mona H. C. Biermann · Benjamin M. Häberle · Jan Multmeier • \\ Maximilian Reinhardt
}

Received: April 22, 2020 / Published online: June 4, 2020

(c) The Author(s) 2020

\section{ABSTRACT}

Introduction: Hidradenitis suppurativa (HS) is a chronic, debilitating, and inflammatory skin disease. The epidemiology of HS varies greatly, with an estimated prevalence ranging from

Digital Features To view digital features for this article go to https://doi.org/10.6084/m9.figshare.12334253.

Electronic supplementary material The online version of this article (https://doi.org/10.1007/s13555$020-00401-y)$ contains supplementary material, which is available to authorized users.

A. Pinter $(\square)$

Clinic for Dermatology, Venereology and

Allergology, University Hospital Frankfurt,

Frankfurt am Main, Germany

e-mail: andreas.pinter@kgu.de

\section{G. Kokolakis}

Psoriasis Research and Treatment Center, Clinic of Dermatology, Venereology, and Allergology and Institute of Medical Immunology, Charité-

Universitätsmedizin Berlin, Berlin, Germany

J. Rech

Department of Internal Medicine, Rheumatology and Immunology, Universitätsklinikum Erlangen, Erlangen, Germany

M. H. C. Biermann · B. M. Häberle

Novartis Pharma GmbH, Nürnberg, Germany

J. Multmeier

Elsevier Health Analytics, Berlin, Germany

M. Reinhardt

Novartis Pharma AG, Basel, Switzerland
$0.03 \%$ to $4 \%$ worldwide. Similar to psoriasis (PsO), HS also exhibits a systemic inflammatory nature with a spectrum of systemic comorbidities. A large health insurance claims (HICs) database is analyzed to determine the demography and epidemiology of $\mathrm{HS}, \mathrm{PsO}$, and $\mathrm{HS}$ with concurrent $\mathrm{PsO}$ (HS-PsO) patients. Furthermore, the comorbidity profiles, including the comorbidity risk of these patient populations, are analyzed.

Methods: This is a noninterventional retrospective analysis of anonymized HICs data using a subset of the Institute of Applied Health Research Berlin (InGef) database. The primary outcome is the prevalence and incidence of HS, $\mathrm{PsO}$, and HS-PsO. Secondary outcomes include comorbidity profiles and a comorbidity risk analysis.

Results: The prevalence and incidence of HS were $0.0681 \%$ and $0.0101 \%$, respectively. The prevalence of HS-PsO was $0.004 \%$ ( $6 \%$ of the total HS population). HS patients frequently suffered from arterial hypertension (45\%), nicotine dependence (46\%), obesity (41\%), and depression (36\%), which were more common in HS-PsO patients compared with HS alone. HS patients had an increased prevalence of metabolic, psychiatric, immune-mediated, and cardiovascular diseases, e.g., overweight/obesity [odds ratio (OR): 2.65, 95\% confidence interval (CI) 2.37-2.96], depression (OR: 1.55, 95\% CI 1.42-1.76), or seronegative rheumatoid arthritis (OR: 2.82, 95\% CI 1.61-4.94) compared with 
the overall population. The increased risk of myocardial infarction in HS patients (OR: 4.1, 95\% CI 3.5-4.8, adjusting for age/sex) was largely attributed to patient's current smoking status (OR: 1.1, 95\% CI 0.8-1.5, adjusting for smoking/age/sex).

Conclusions: HS patients show a broad spectrum of inflammatory and metabolic syndromerelated comorbidities, with an increased risk by concurrent PsO. Important for clinical practice, the elevated cardiovascular risk of HS patients can be largely attributed to smoking.

Keywords: Cardiovascular risk; Comorbidity; Epidemiology; Hidradenitis suppurativa; Psoriasis; Smoking

\section{Key Summary Points}

A health insurance dataset of more than 7 million individuals in Germany was analyzed to determine the epidemiology, comorbidity profiles, and comorbidity risk of hidradenitis suppurativa (HS), psoriasis (PsO), and HS with concomitant PsO.

The prevalence and incidence of HS was 68/100,000 and 10.1/100,000; Approximately $6 \%$ of HS patients had a concomitant diagnosis of $\mathrm{PsO}$.

HS patients showed an increased risk for metabolic, psychiatric, immunemediated, and cardiovascular comorbidities.

HS with concomitant PsO aggravated comorbidity frequencies of HS patients, especially regarding psychiatric diseases.

The increased risk to develop cardiovascular comorbidities in HS patients was largely attributed to smoking.

\section{INTRODUCTION}

Hidradenitis suppurativa (HS) is a chronic, progressive, highly inflammatory, painful, and destructive follicular skin disorder. HS manifests in nodules which can progress to abscesses, sinus tracts, and fistulae [1]. Inflammatory lesions mainly affect the inverse body areas, including axillae, the inguinal and anogenital region, and inframammary areas in women $[2,3]$. Women are more frequently affected than men, with a ratio of 3:1 [4,5]. Due to variations in clinical appearance, treatment response, and involved specialties, accurate diagnosis can be complex and is often delayed [6].

Conflicting opinions exist regarding the epidemiology of HS, with an estimated prevalence ranging from $0.03 \%$ to $4 \%$ worldwide $[7,8]$. The average prevalence has been reported as $0.8 \%$ in the UK [9], $1 \%$ in France, $0.7 \%$ in Australia [10], and $0.1 \%$ in the USA [11]. Scarce data on global incidences of HS are available; a study from the UK reported an annual incidence of physician-diagnosed cases from 1996 to 2013 as $0.028 \%$ [9]; however, in the USA, an overall incidence of $0.011 \%$ was reported between 2015 and 2016 [11].

In comparison with other chronic cutaneous diseases such as psoriasis (PsO), expenses for HS disease management are dominated by highcost settings, e.g., inpatient and emergency department care, including surgical interventions [12]. In addition to the severe impact on a patient's quality of life (QoL), HS impairs the patient's ability to work, subsequently affecting annual income, resulting in both unmet medical and socioeconomic needs [13, 14].

To provide optimal health care to HS patients and to quantify the societal burden of this disease, it is essential to understand the demography and epidemiology of HS, frequently associated comorbidities, and risk factors. Analysis of the comorbidity profile and risk are important to understand and dissect the systemic aspects of the disease and can help to optimize clinical care.

Smoking has been attributed to HS development and has been identified as a decisive risk 
factor, with the incidence of HS doubled amongst smokers compared with nonsmokers $[5,15,16]$. HS is often accompanied by severe comorbidities, including metabolic syndrome and Crohn's disease (CD) $[6,17]$. In addition, patients with HS have a higher prevalence of depression, anxiety, and suicidal ideation, demonstrating the impact of HS on psychosocial wellbeing and QoL $[18,19]$.

HS and PsO have overlapping immunopathogenic pathways as well as patterns of inflammation-related comorbidity [20]. Data on co-occurrence of both pathologies are however scarce. Type 1 and 3 cytokines such as TNF- $\alpha$, Interferon- $\gamma$, IL-1- $\alpha / \beta$, IL- 6 , IL- 8 , and IL-17 are upregulated, indicating that the Th1/Th17 axis is activated and may drive inflammation in both diseases [21, 22].

A large-scale population study in Israel reported a greater prevalence of $\mathrm{HS}$ in $\mathrm{PsO}$ patients than in the general population. HS and $\mathrm{PsO}$ coexistence was associated with an increased prevalence of obesity and smoking [23]. A Danish monocentric analysis of patients with HS and PsO consistently observed a threefold increased risk of HS patients for concomitant PsO [24]; other studies suggest more severe comorbidities in concomitant HS and $\mathrm{PsO}[25]$.

The present study is conducted to assess the demography and epidemiology of HS patients, PsO patients, and HS patients with concurrent $\mathrm{PsO}$ in the German population. In addition, comorbidity profiles are assessed, and a risk analysis is conducted for metabolic, cardiovascular, psychiatric, and immunological comorbidities.

\section{METHODS}

\section{Study Design and Data Source}

Approximately $85 \%$ of the German population ( $\sim 70$ million individuals) are insured by the statutory health insurance (SHI) system, which includes approximately 120 independent health insurance companies. This is a noninterventional retrospective analysis conducted using health insurance claims (HICs) data from the Forschungsdatenbank des InGef - Institut für angewandte Versorgungsforschung Berlin $\mathrm{GmbH}$ (InGef) research database. InGef is a complete longitudinal claims dataset of approximately 7 million individual patients. InGef includes data from different health care sectors ranging from outpatient care, hospital treatment, and the pharmacy sector to data about physiotherapy and technical aids. The analyses were completed on a subset of 4.1 million patients stratified by age and sex based on the population structure of Germany in 2013 (German Federal Statistical Office DeStatis), which allows extrapolation of these epidemiological findings to the overall German adult population.

All patient-level data in the InGef research database were deidentified to comply with data protection regulations. Use of the study database for health services research is therefore fully compliant with German federal law, and accordingly, ethical approval was not needed.

\section{Study Population}

The observational period spanned from January 1, 2012 to December 31, 2017. Adult patients ( $\geq 18$ years) who were continually insured without interruption within the above time frame and with a diagnosis of $\mathrm{HS}$ and/or PsO were included in this study. Diagnosis could be a hospital main secondary discharge or outpatient diagnosis verified in at least two quarters within one rolling year (M2Q criterion). Patients with $\mathrm{HS}$ and $\mathrm{PsO}$ were identified using the International Statistical Classification of Diseases and Related Health Problems (ICD) codes (10th revision, German modification; L73.2: hidradenitis suppurativa, L40.0: plaque psoriasis).

\section{Epidemiological Measures}

HS prevalence was classified as having a documented HS diagnosis (M2Q) between January 1, 2012 and December 31, 2016. Similarly, PsO prevalence was classified as having a diagnosis of PsO with no concomitant HS (M2Q) in the same time period. Prevalence of HS with concurrent PsO (HS-PsO) was classified as having a 
diagnosis of HS and PsO (M2Q) between January 1, 2012 and December 31, 2016. Incidence of either HS, PsO, or HS-PsO was defined by a diagnosis of HS, PsO, or HS-PsO between January 1, 2017 and December 31, 2017 with no prior diagnosis of $\mathrm{HS}, \mathrm{PsO}$, or $\mathrm{HS}-\mathrm{PsO}$, respectively, between January 1, 2012 and December 31,2016 . The incidence and prevalence of HS, $\mathrm{PsO}$, and HS-PsO were calculated by dividing the total number of diagnosed patients by the total number of adults in the database within the specified period.

\section{Patient Characteristics and Comorbidity Profiles}

Secondary objectives of this study include examining patient demographics (age and sex), diagnosing specialties, comorbidity profiles (frequently documented diagnoses on ICD-10 GM three-digit level relevant to HS), and risk of comorbidity development.

Comorbidities were grouped into four main categories: metabolic, psychiatric, cardiovascular, and autoimmune/immune related. The frequency of comorbidities in HS, PsO, and HS-PsO were compared with a reference population insured by the SHI system in Germany. To analyze the comorbidity risk, HS, PsO, and HS$\mathrm{PsO}$ patients were age and sex matched to the SHI German population at a ratio of 1:4. For each patient of each target population, four individuals both age and sex matched with no diagnosis of PsO or HS were selected from the database of SHI individuals as healthy controls (control population). Analogous to the patient cohort, only patients who were continually insured from January 1, 2012 to December 31, 2017 and $\geq 18$ years were included. As smoking is highly prevalent among HS patients and can have an impact on certain comorbidities, risk analysis was carried out in matched as well as unmatched for tobacco abuse/nicotine dependence (smoking status) [16, 26]. This enabled the assessment of the impact of smoking on comorbidity risk. Parameters calculated include the odds-ratio (OR), 95\% confidence interval (CI), and p-values for each individual comorbidity compared with the SHI German population.

\section{Data Analysis}

SAS Enterprise Guide software (version 9.2) was used for statistical programming. All HS and PsO patients fulfilling the inclusion criteria were included in the analyses to get representative results for the German population. The estimation of the overall SHI German population was based on the official statistics of individuals covered by SHI in the respective year (https://www.gbe-bund.de). The ratio of insured individuals in the official statistics to patients found in the age and sex stratified analytical database was used as a factor to extrapolate the total number of individuals under SHI coverage. This factor was derived from the following formula:

$$
\text { Extrapolation Factor }=\frac{N_{\text {SHI }}}{N_{\text {Database }}},
$$

where $N_{\mathrm{SHI}}$ represents the total number of individuals enrolled in SHI and $N_{\text {Database }}$ represents the total number of individuals in the research database.

A factor of 20.16 was used to extrapolate results to the SHI population.

\section{RESULTS}

\section{Patient Characteristics}

The prevalence and incidence were 68.1 cases per 100,000 and 10.1 cases per 100,000 per year for HS and 1962.9 cases per 100,000 and 96.6 cases per 100,000 per year for PsO, respectively. Although the prevalence and incidence of HSPsO were generally low (4.3 cases per 100,000 and 0.2 cases per 100,000 per year), this accounted for approximately $6.3 \%$ and $1.9 \%$ of the HS population, respectively (Table 1 ). Men and women were affected equally in the analyzed incident and prevalent population (Supplementary Table 1). Compared with $\mathrm{PsO}, \mathrm{HS}$ was more frequently diagnosed in a hospital (PsO, 8.5\%; HS, 43.8\%), whereas $\mathrm{PsO}$ was 
Table 1 Incidence and prevalence of $\mathrm{HS}, \mathrm{PsO}$, and HS$\mathrm{PsO}$ cohorts

\begin{tabular}{lll}
\hline Group & $\begin{array}{l}\text { Prevalence }^{\mathbf{a}} \\
\text { Cases per 100,000 }\end{array}$ & $\begin{array}{l}\text { Incidence }^{\mathbf{b}} \\
\text { Cases per 100,000 }\end{array}$ \\
\hline $\mathrm{HS}$ & 68.1 & 10.1 \\
$\mathrm{PsO}$ & 1962.9 & 96.6 \\
$\mathrm{HS}-\mathrm{PsO}$ & 4.3 & 0.2 \\
\hline
\end{tabular}

${ }^{a}$ Population includes patients with a diagnosis of HS, $\mathrm{PsO}$, or both between January 1, 2012 and December 31, 2016; ${ }^{\text {b }}$ Population includes patients with a new diagnosis of HS, PsO, or both between January 1, 2017 and December 31, 2017; HS, hidradenitis suppurativa; $\mathrm{PsO}$, psoriasis; HS-PsO, hidradenitis suppurativa with concurrent psoriasis

generally diagnosed by a dermatologist (PsO, $60.3 \%$; HS, 22.9\%) or a general practitioner (PsO, 16.6\%; HS, 9.4\%) (data on file).

The majority of HS patients $(70 \%)$ were $\leq 49$ years with only $2.6 \% \geq 70$ years (median age: 43.0 years) (Fig. 1a). Conversely, the PsO population tended to be older, with $69.5 \%$ of PsO patients $\geq 50$ years and $24.4 \% \geq 70$ years (median age: 58.0 years) (Fig. $1 \mathrm{~b}$ ). The ages of HS-PsO patients closely followed the trend of those with HS alone, with a predominance of younger patients and only $5.2 \%$ of patients being $\geq 70$ years (median age: 47.0 years) (Fig. 1c).

\section{Frequent Comorbidities}

The most common comorbidity in HS patients was nicotine dependence, with almost half of the population affected within the HS and HS$\mathrm{PsO}$ cohorts (45.7\% and $48.4 \%$, respectively), compared with $16 \%$ of $\mathrm{PsO}$ patients and $11.7 \%$ in the SHI German control population. Metabolic and cardiovascular comorbidities such as obesity (40.8\% in $\mathrm{HS}, 31.4 \%$ in $\mathrm{PsO}, 47.4 \%$ in HS-PsO, and $21.4 \%$ in control population) and type 2 diabetes $(18.6 \%$ in $\mathrm{HS}, 24.1 \%$ in $\mathrm{PsO}$, $21.1 \%$ in $\mathrm{HS}-\mathrm{PsO}$, and $16.4 \%$ in control population) were frequent in both $\mathrm{HS}$ and $\mathrm{PsO}$ patients, and more frequent compared with the control population. Although many comorbidities were more common in $\mathrm{PsO}$ compared with HS [e.g., arterial hypertension (44.7\% in HS, 64.3\% in PsO, 50.5\% in HS-PsO, and $50.5 \%$ in control population), ischemic heart disease $(9.9 \%$ in $\mathrm{HS}, 21.5 \%$ in $\mathrm{PsO}, 16.8 \%$ in $\mathrm{HS}-\mathrm{PsO}$, and $14.8 \%$ in control population), and angina pectoris $(4.1 \%$ in $\mathrm{HS}, 8.1 \%$ in $\mathrm{PsO}$, $9.5 \%$ in $\mathrm{HS}-\mathrm{PsO}$, and $5.4 \%$ in control population)], the frequency of Crohn's disease (3.4\% in $\mathrm{HS}, 1.2 \%$ in $\mathrm{PsO}$, N/A in HS-PsO, and $0.7 \%$ in control population) and anxiety (20.2\% in HS, $15.1 \%$ in $\mathrm{PsO}, 14.7 \%$ in $\mathrm{HS}-\mathrm{PsO}$, and $12.4 \%$ in control population) were more common in $\mathrm{HS}$ patients.

More patients with HS-PsO suffered from psychiatric diseases, such as major depressive disorder (single or recurrent; $35.9 \%$ in $\mathrm{HS}$, $32.4 \%$ in $\mathrm{PsO}, 46.3 \%$ in $\mathrm{HS}-\mathrm{PsO}$, and $25.9 \%$ in control population), somatoform disorders (36.0\% in $\mathrm{HS}, 33.5 \%$ in $\mathrm{PsO}, 44.2 \%$ in $\mathrm{HS}-\mathrm{PsO}$, and $26.8 \%$ in control population), and phobic anxiety disorders $(5.3 \%$ in $\mathrm{HS}, 3.7 \%$ in $\mathrm{PsO}$, $6.3 \%$ in $\mathrm{HS}-\mathrm{PsO}$, and $2.9 \%$ in control population). In addition to psychological burden, the presence of both HS and PsO resulted in a higher frequency of rheumatological conditions, such as enteropathic arthropathies $(0.6 \%$ in $\mathrm{HS}, 11.4 \%$ in $\mathrm{PsO}, 19.0 \%$ in $\mathrm{HS}-\mathrm{PsO}$, and $0.2 \%$ in control population), other inflammatory spondylopathies, and rheumatoid arthritis (3.4\% in $\mathrm{HS}, 9.8 \%$ in $\mathrm{PsO}, 14.7 \%$ in $\mathrm{HS}-\mathrm{PsO}$, and $3.2 \%$ in control population). Furthermore, HS$\mathrm{PsO}$ patients showed a clear increase in coprevalence of obesity (40.8\% in HS, $31.4 \%$ in $\mathrm{PsO}, 47.4 \%$ in $\mathrm{HS}-\mathrm{PsO}$, and $21.4 \%$ in control population), myocardial infarction (2.3\% in HS, $4.3 \%$ in $\mathrm{PsO}, 6.3 \%$ in $\mathrm{HS}-\mathrm{PsO}$, and $3.1 \%$ in control population), or atherosclerosis (8.5\% in HS, $15.6 \%$ in PsO, $11.6 \%$ in HS-PsO, and $10.0 \%$ in control population) (Table 2 ).

\section{Comorbidity Risk}

HS patients had a greater risk for immune-mediated comorbidities, such as seronegative rheumatoid arthritis (OR: 2.8, 95\% CI 1.6-4.9, $p<0.0005$ ) and type 1 diabetes (OR: 2.2, 95\% CI $1.7-2.9, p<0.0001)$. In addition, HS patients had a greater risk for metabolic diseases, such as 
A

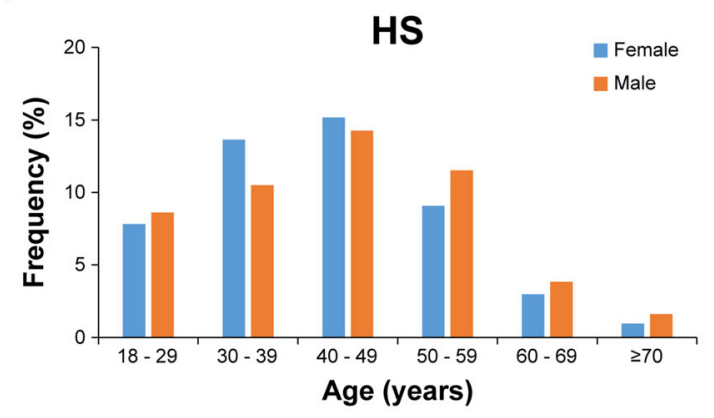

C

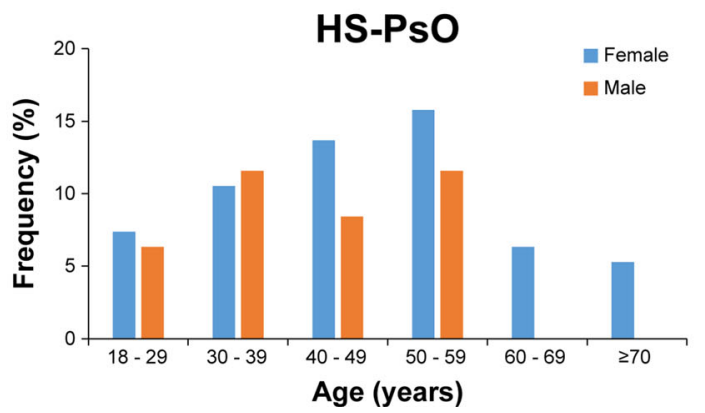

Fig. 1 Age and sex distribution of $\mathrm{HS}, \mathrm{PsO}$, and $\mathrm{HS}-\mathrm{PsO}$ cohorts. Bar graphs showing percentage (\%) of patients within specified age groups with HS (female, $n=870$; male, $n=882$; a), $\mathrm{PsO}$ (female, $n=24,238$; male, $n=27,409 ; \mathbf{b}$ ), and HS-PsO (female, $n=56$; male,

type 2 diabetes (OR: 2.1, 95\% CI 1.8-2.4, $p<0.0001$ ) and obesity (OR: 2.7, 95\% CI 2.4-3.0, $p<0.0001)$, compared with an age-, sex- and nicotine dependence (current ICDcoding status)-matched control population (Fig. 2a). When HS and SHI German control populations were unmatched for nicotine dependence, the risk of developing cardiovascular comorbidities increased dramatically (e.g., myocardial infarction, OR: 1.1, 95\% CI 0.8-1.5 in nicotine dependent-matched compared with OR: 4.1, 95\% CI 3.45-4.75 in nicotine dependent-unmatched populations), demonstrating the underlying impact of smoking on cardiovascular risk (Fig. 2a, Supplementary Fig. 1A). In contrast, the risk for metabolic and immunemediated comorbidities was not modulated by smoking status. Similar risks were identified in HS cohorts both matched and unmatched for nicotine dependence [e.g., type 2 diabetes in matched (OR: 2.1, 95\% CI 1.8-2.4) versus
B

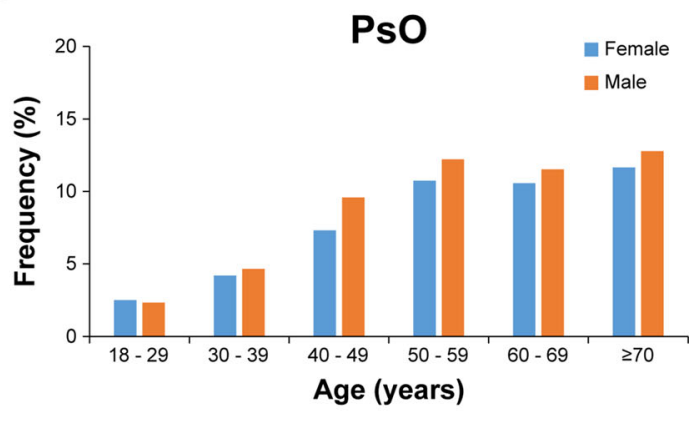

$n=39 ;$ c) in German population between January 1, 2012 and December 31, 2017. HS hidradenitis suppurativa, $P s O$ psoriasis, $H S-P s O$ hidradenitis suppurativa with concurrent psoriasis

unmatched (OR: 2.3, 95\% CI 2.26-2.30) and other rheumatoid arthritis in matched (OR: 1.8, 95\% CI 1.3-2.4) versus unmatched (OR: 1.9, 95\% CI 1.7-2.2)] (Fig. 2a, Supplementary Fig. 1A).

Consistent with the high overall prevalence of smoking, HS patients have an increased risk of mental and behavioral disorders due to the use of tobacco (OR: 1.6, 95\% CI 1.4-1.9, $p<0.0001)$. In addition, HS patients have a significantly increased risk for major depressive disorders (recurrent, OR: 1.6, 95\% CI 1.3-1.8, $p<0.0001$; single episode, OR: $1.6,95 \%$ CI 1.4-1.7, $p<0.0001$ ) (Fig. 2a).

Similar to HS, PsO patients had an increased risk of developing psychiatric and metabolic comorbidities compared with the SHI German control population (Fig. 2b), although the risk in $\mathrm{PsO}$ patients was numerically lower compared with HS patients (e.g., type 2 diabetes, OR: $2.1,95 \%$ CI 1.8-2.4 in HS versus OR: 1.3, 

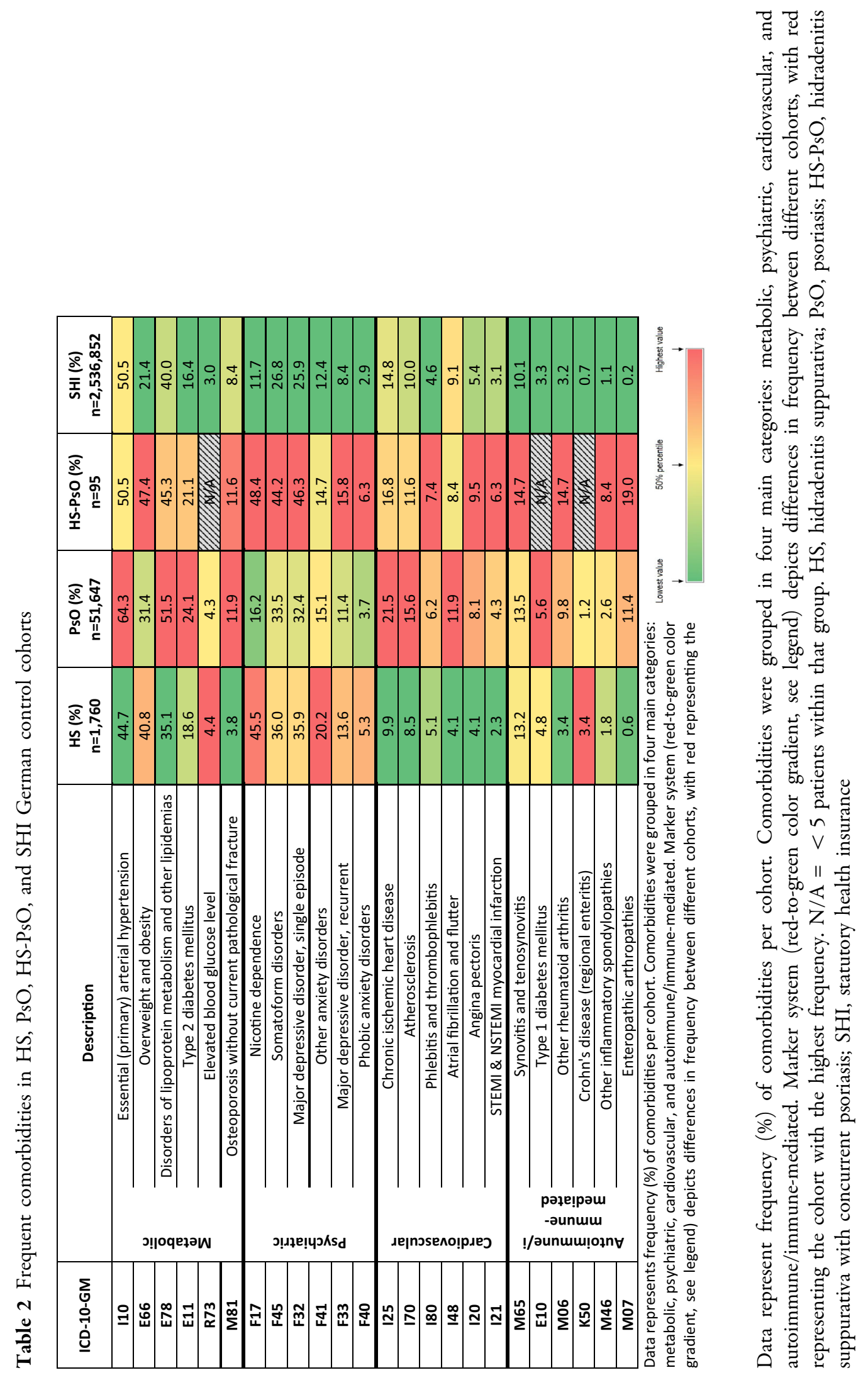
A

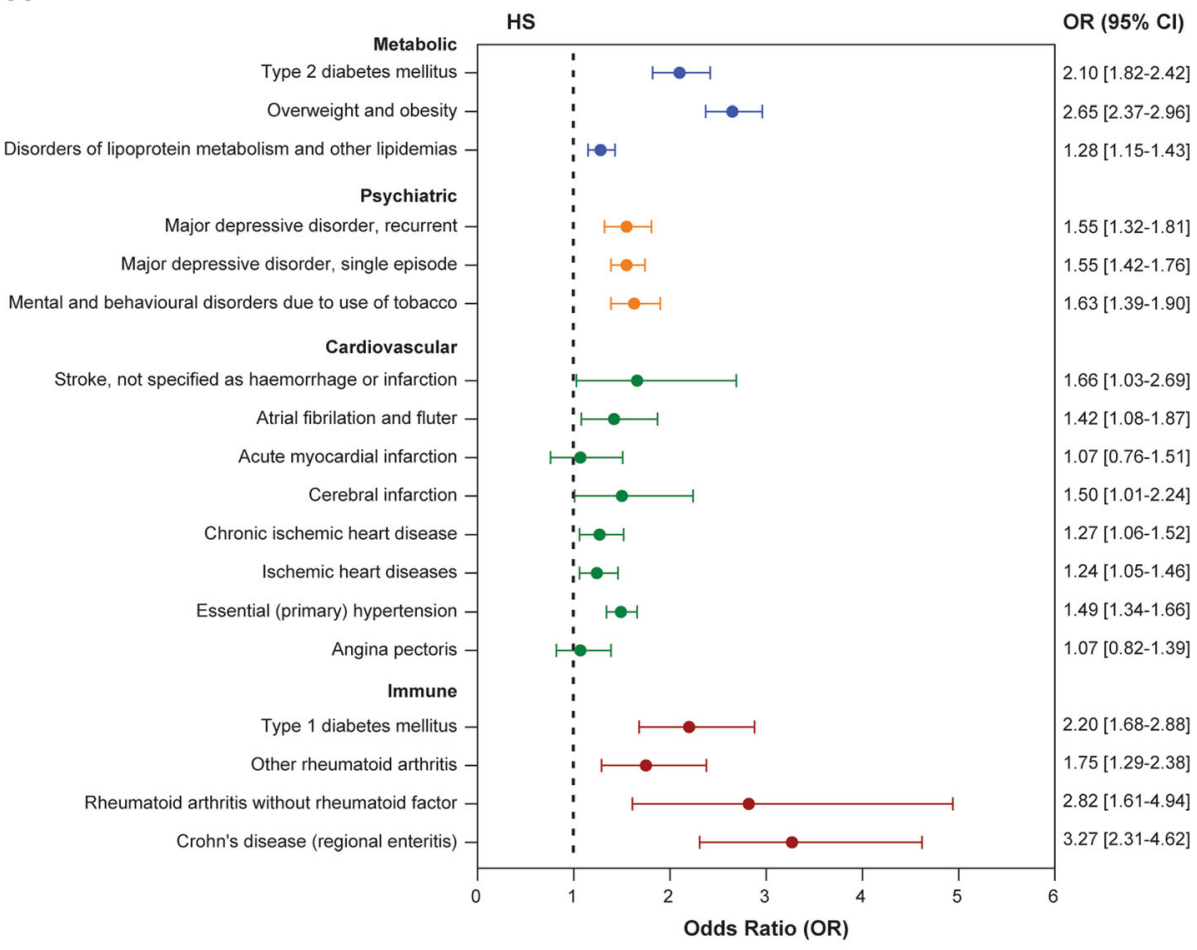

B

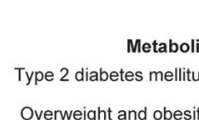

Disorders of lipoprotein metabolism and other lipidemias

Psychiatric

Major depressive disorder, recurrent

Major depressive disorder, single episode

Mental and behavioural disorders due to use of tobacco

Cardiovascular

Stroke, not specified as haemorrhage or infarction

Atrial fibrilation and fluter

Acute myocardial infarction

Cerebral infarction

Chronic ischemic heart disease

Ischemic heart diseases

Essential (primary) hypertension

Angina pectoris

Immune

Type 1 diabetes mellitus

Other rheumatoid arthritis

Rheumatoid arthritis without rheumatoid factor

Crohn's disease (regional enteritis)
$\mathrm{PsO}$

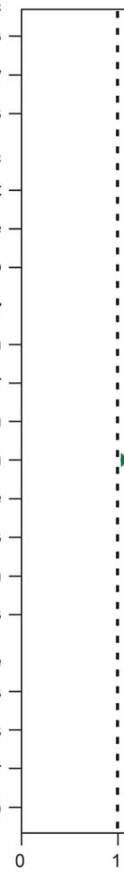

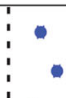

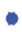

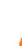

.

(1)

|el

:

$\mid$

(1)

101

ar

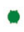

-

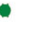

-

$\bullet$

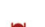

|

ror

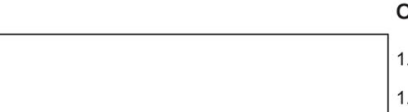

1.33 [1.30-1.36]

1.50 [1.47-1.53]

$1.24[1.21-1.26]$

1.38 [1.34-1.42]

$1.34[1.31-1.37]$

1.37 [1.31-1.44]

$1.21[1.14-1.28]$

1.18 [1.15-1.22]

1.20 [1.15-1.26]

1.09 [1.04-1.14]

1.29 [1.26-1.32]

1.28 [1.26-1.31]

1.33 [1.30-1.36]

1.29 [1.25-1.34]

1.45 [1.39-1.52]

2.89 [2.78-3.00]

2.80 [2.59-3.02]

1.71 [1.55-1.87] 
4Fig. 2 Risk analysis of selected comorbidities in HS and $\mathrm{PsO}$ cohorts matched for age, sex, and nicotine dependence. Forest plots demonstrating risk analysis of common comorbidities in hidradenitis suppurativa (HS, $n=1752$; a) and psoriasis ( $\mathrm{PsO}, n=51,647$; b) patients compared with the SHI German population. Patients were matched for age, sex, and nicotine dependence to the SHI German population at a ratio of 1:4. Data are displayed as odds ratio (OR), and error bars represent the $95 \%$ confidence interval values (95\% CI). Comorbidities are categorized as metabolic (blue), psychiatric (orange), cardiovascular (green), and immune related (red). HS hidradenitis suppurativa, $P s O$ psoriasis, $H S-P s O$ hidradenitis suppurativa with concurrent psoriasis

95\% CI 1.3-1.4 in PsO). Smoking was also identified as an underlying modulator of cardiovascular comorbidities (but not metabolic or chronic inflammation-related comorbidities) in PsO patients, as the risk was sufficiently reduced in populations matched for nicotine dependence (Fig. 2b, Supplementary Fig. 1B). The impact of smoking on cardiovascular risk was, however, smaller in PsO compared with HS patients. A diagnosis of PsO increased the risk of rheumatic diseases, consistent with psoriatic arthritis (PsA) being reported as the most prevalent comorbidity of $\mathrm{PsO}$ patients [27]. Due to the small sample size of the HS-PsO population $(n=95)$, the OR analysis could not deliver conclusive results.

\section{DISCUSSION}

This study describes the demography and epidemiology of HS, PsO, and HS-PsO. Here, we show that most HS patients were between the ages of 30 and 60 years. In studies from both the UK and the USA, HS prevalence peaked in patients around 40 years, similar to this German population $[11,28]$. The age distribution of $\mathrm{PsO}$ validated a previous study of the German population, in which a large proportion of the population were over 50 years [29]. Consistent with recent reports, HS-PsO patients had a similar age distribution to HS patients, with the majority between 30 and 60 years [23]. In contrast to the sex bias reported by smaller case-control studies, the sex ratio in the cohorts diagnosed with $\mathrm{HS}, \mathrm{PsO}$, and $\mathrm{HS}-\mathrm{PsO}$ in the present study was balanced (Supplementary Table 1) $[4,5]$.

Interestingly, the percentage of patients with HS with or without PsO declined after the sixth decade of life. Although previously described in epidemiological studies, the immunological reasons for this are not well understood. However, women regularly report the onset of HS disease with menarche, flare up according to the menstrual cycle, and resolution of disease in later life following menopause, supporting the idea of hormonal influence in HS $[28,30]$.

The prevalence and incidence of HS in this study were relatively low (68.1 cases per 100,000 and 10.1 cases per 100,000 per year, respectively) compared with figures reported worldwide. The prevalence and incidence of HS varies greatly depending on study design, population size, and determination of diagnosis. HICs data and electronic health records in large populations have yielded prevalences of $0.1 \%$ [11] (100 cases per 100,000) and 0.05\% [31] (50 cases per $100,000)$, respectively, whereas smaller self-reported studies using questionnaires observed values of $4 \%$ [32] (4000 cases per 100,000) and $2.1 \%$ [33] (2100 cases per 100,000), respectively. Differences in reported prevalence can be related to a higher misdiagnosis of HS in clinical routine and, therefore, underreporting in SHI. Nevertheless, the observed incidence of HS in this study was in line with other larger studies [34]. The prevalence of $\mathrm{PsO}, 2 \%$ (1962.9 cases per 100,000), in our study was consistent with others, validating this study population $[35,36]$. Although the prevalence and incidence of HS-PsO were generally low, these figures account for $6.3 \%$ and $1.9 \%$ of the HS population, respectively. Most HS patients were diagnosed in a hospital, whereas PsO patients were generally diagnosed in private practices, suggesting that the observed cases of HS may be low due to a lack of identification of disease/delayed diagnosis. The bias of delayed diagnosis was determined to be on average 1.6 years for PsO but 7.2 years for HS [37]. The main reasons for delayed diagnosis of HS are a lack of knowledge as well as the heterogeneous phenotype and unspecific early signs of the disease [38]. 
Consistent with previous reports, HS patients showed a higher prevalence and increased risk for the development of cardiovascular and metabolic comorbidities compared with the control population (Fig. 2a) [39, 40]. This was also true, albeit to a smaller extent, for PsO patients (Fig. 2b). Intriguingly, in the HS population, the cardiovascular risk was primarily determined by smoking status (Fig. 2a versus Supplementary Fig. 1A). Interestingly, matching for nicotine dependence reduced the risk of myocardial infarction in HS patients almost to the level of the control population. In the PsO population, the risk of developing these comorbidities was both generally lower and less modulated by smoking status (Fig. $2 b$ versus Supplementary Fig. 2B). This indicates that the increased risk of cardiovascular events in HS patients is largely attributed to smoking and not to HS-related processes, e.g., systemic inflammatory processes caused by HS [41, 42]. This is of high relevance for clinical practice since the prevalence of smoking is exceptionally high among HS patients. In this analysis, almost half of the HS population were nicotine dependent compared with $16.2 \%$ of PsO patients and $11.7 \%$ of the overall population. Smoking cessation does not only improve treatment outcomes in HS but also strongly reduces the elevated cardiovascular risk in these patients [43].

HS-PsO patients suffer with more psychological and rheumatological comorbidities compared with $\mathrm{HS}$ or $\mathrm{PsO}$ alone patients. Although it is well reported that $\mathrm{HS}$ and $\mathrm{PsO}$ patients have a reduced QoL and higher prevalence of depression, there are limited data on the effect of HS-PsO on psychological comorbidities [23, 25, 44, 45]. Consistently, Patel et al. described a higher proportion of HS-PsO patients with depression compared with HS or $\mathrm{PsO}$ alone [25]. The current study is the first to describe the increased frequency of rheumatological comorbidities in a HS-PsO population. Here, we observed a fourfold increase in the frequency of inflammatory spondyloarthropathies in HS-PsO compared with HS alone. This increase may resemble the pathomechanistic influence of $\mathrm{PsO}$, with studies demonstrating that the risk of PsA is constant over time following a diagnosis of $\mathrm{PsO}$, with a $1 \%$ mean annual incidence of PsA in $\mathrm{PsO}$ patients [46, 47].

The key strength of HIC analyses is the very large sample size of hospitalized as well as outpatient-treated continuously insured patients in Germany. This allows for analysis of the epidemiology of PsO and HS coprevalence. However, mild to moderate HS forms are commonly misdiagnosed, especially at the beginning of the disease course; thus, HIC data may rather underestimate the prevalence of HS [48]. This may be also impacted by the fact that HS was not exclusively diagnosed by dermatologists, e.g., in case of HS diagnosis in the hospital. Prevalence of HS calculated from other German SHI data showed similar incidence and prevalence and estimated the rate of potential misdiagnosis of HS at 34.7\% [49]. The analyzed HIC data do not allow assessment of the influence of ethnicity, the patient's profession, educational level, subtypes of HS, laboratory data, or the duration or severity of both diseases on the prevalence of different comorbidities. Coding inconsistencies as well as ICD-10 (L73.2) coding error by misdiagnosis (mainly of HS) might further contribute to lower numbers in HIC analysis. Correct diagnosing is, however, more reliable compared with patient-completed questionnaires, which is noteworthy with phenotypically complex diseases such as HS. HS was mostly diagnosed in a hospital setting, with only one-fifth of patients diagnosed by dermatologists. This may result in an overrepresentation of patients with high disease severity within our population. Analyses of comorbidity frequency and risk were performed independently of disease severity, not visible in HIC data, therefore not allowing for a correlation of comorbidity profile/risk and disease activity. Although this analysis was conducted using a HICs dataset restricted to Germany, its findings may be valuable in other countries with a similar demographic structure, especially within Europe.

In summary, patients with chronic inflammatory skin diseases such as $\mathrm{PsO}$ and HS have an increased occurrence of comorbidity. Interestingly, the prevalence of concomitant psychiatric and cardiovascular diseases is even 
more increased upon co-occurrence of PsO and HS (HS-PsO cohort). The elevated cardiovascular risk of HS patients can, however, be largely attributed to smoking.

\section{ACKNOWLEDGEMENTS}

Funding. This analysis and the journal's Rapid Service Fee were funded by Novartis Pharma GmbH, Nürnberg, Germany.

Medical Writing Assistance. The authors thank Trudy McGarry, PhD and Jackie L. Johnson, PhD of Novartis Ireland Ltd. for providing medical writing support/editorial support, which was funded by Novartis Pharma GmbH Germany, in accordance with Good Publication Practice (GPP3) guidelines (https://www.ismpp. org/gpp3).

Authorship. All named authors meet the ICMJE criteria for authorship for this article, take responsibility for the integrity of the work as a whole, and have given their approval for this version to be published.

Disclosures. Andreas Pinter has worked as an investigator and/or speaker and/ or advisor for the following pharmaceutical companies: AbbVie, Almirall-Hermal, Amgen, Biogen Idec, Boehringer-Ingelheim, Celgene, GSK, Eli-Lilly, Galderma, Hexal, Janssen, LEOPharma, MC2, Medac, Merck Serono, Mitsubishi, MSD, Novartis, Pascoe, Pfizer, Tigercat Pharma, Regeneron, Roche, Sandoz Biopharmaceuticals, Sanofi-Genzyme, Schering-Plough und UCB Pharma. Georgios Kokolakis has worked as an investigator and/or speaker and/or advisor for the following pharmaceutical companies: AbbVie Deutschland GmbH \& Co. KG, Abbott GmbH, Actelion Pharmaceuticals Ltd., Basilea Pharmaceutica Ltd., Bayer AG, Biogen IDEC GmbH, Celgene $\mathrm{GmbH}$, Janssen-Cilag GmbH, LEO Pharma GmbH, Lilly Deutschland $\mathrm{GmbH}$, MSD Sharp \& Dohme GmbH, Novartis Pharma GmbH, Parexel International $\mathrm{GmbH}$, Pfizer Deutschland GmbH, and UCB Pharma $\mathrm{GmbH}$. Juergen Rech has worked as an investigator and/or speaker and/or advisor for the following pharmaceutical companies: AbbVie, Amgen, Biogen Idec, Boehringer-Ingelheim, Celgene, Chugai, GSK, Eli-Lilly, Hexal, Janssen, Medac, Merck Serono, MSD, Novartis, Pfizer, Roche, Sandoz Biopharmaceuticals, Sanofi-Genzyme, Schering-Plough und UCB Pharma. Jan Multmeier is an employee of Elsevier Health Analytics, Berlin, Germany. Mona H. C. Biermann and Benjamin M. Häberle are employees of Novartis Pharma GmbH, Nürnberg, Germany. Maximilian Reinhardt is an employee of Novartis Pharma AG, Basel, Switzerland.

Compliance and Ethics Guidelines. All patient-level data in the InGef research database were de-identified to comply with data protection regulations. Use of the study database for health services research is therefore fully compliant with German federal law, and accordingly, ethical approval was not needed.

Data Availability. Novartis is committed to sharing with qualified external researchers, access to patient-level data and supporting clinical documents from eligible studies. These requests are reviewed and approved by an independent review panel on the basis of scientific merit.

Open Access. This article is licensed under a Creative Commons Attribution-NonCommercial 4.0 International License, which permits any non-commercial use, sharing, adaptation, distribution and reproduction in any medium or format, as long as you give appropriate credit to the original author(s) and the source, provide a link to the Creative Commons licence, and indicate if changes were made. The images or other third party material in this article are included in the article's Creative Commons licence, unless indicated otherwise in a credit line to the material. If material is not included in the article's Creative Commons licence and your intended use is not permitted by statutory regulation or exceeds the permitted use, you will need to obtain permission directly from the copyright holder. To view a copy of this licence, 
visit http://creativecommons.org/licenses/by$\mathrm{nc} / 4.0 /$.

Open Access. This article is licensed under a Creative Commons Attribution-NonCommercial 4.0 International License, which permits any non-commercial use, sharing, adaptation, distribution and reproduction in any medium or format, as long as you give appropriate credit to the original author(s) and the source, provide a link to the Creative Commons licence, and indicate if changes were made. The images or other third party material in this article are included in the article's Creative Commons licence, unless indicated otherwise in a credit line to the material. If material is not included in the article's Creative Commons licence and your intended use is not permitted by statutory regulation or exceeds the permitted use, you will need to obtain permission directly from the copyright holder. To view a copy of this licence, visit http://creativecommons.org/licenses/by$\mathrm{nc} / 4.0 /$.

\section{REFERENCES}

1. Dufour DN, Emtestam L, Jemec GB. Hidradenitis suppurativa: a common and burdensome, yet under-recognised, inflammatory skin disease. Postgrad Med J. 2014;90(1062):216-21.

2. Jansen I, Altmeyer P, Piewig G. Acne inversa (alias hidradenitis suppurativa). J Eur Acad Dermatol Venereol. 2001;15(6):532-40.

3. Poli F, Jemec GBE, Revuz J. Clinical presentation. In: Revuz J, Leyden JJ, editors. Hidradenitis suppurativa. Berlin: Springer; 2006. p. 11-24.

4. Jemec GB. Hidradenitis suppurativa. J Cutan Med Surg. 2003;7(1):47-56.

5. Revuz JE, Canoui-Poitrine F, Wolkenstein P, Viallette C, Gabison G, Pouget F, et al. Prevalence and factors associated with hidradenitis suppurativa: results from two case-control studies. J Am Acad Dermatol. 2008;59(4):596-601.

6. Hessam S, Scholl L, Sand M, Schmitz L, Reitenbach S, Bechara FG. A novel severity assessment scoring system for hidradenitis suppurativa. JAMA Dermatol. 2018;154(3):330-5.
7. Jemec GB, Heidenheim M, Nielsen NH. The prevalence of hidradenitis suppurativa and its potential precursor lesions. J Am Acad Dermatol. 1996;35(2 Pt 1):191-4.

8. Naldi L. Epidemiology. In: Revuz J, Leyden JJ, editors. Hidradenitis suppurativa. Berlin: Springer; 2006. p. 58-64.

9. Ingram JR, Jenkins-Jones S, Knipe DW, Morgan CLI, Cannings-John R, Piguet V. Population-based clinical practice research Datalink study using algorithm modelling to identify the true burden of hidradenitis suppurativa. $\mathrm{Br} \mathrm{J}$ Dermatol. 2018;178(4):917-24.

10. Calao M, Wilson JL, Spelman L, Billot L, Rubel D, Watts AD, et al. Hidradenitis Suppurativa (HS) prevalence, demographics and management pathways in Australia: a population-based cross-sectional study. PLoS ONE. 2018;13(7):e0200683.

11. Garg A, Kirby JS, Lavian J, Lin G, Strunk A. Sex- and age-adjusted population analysis of prevalence estimates for hidradenitis suppurativa in the United States. JAMA Dermatol. 2017;153(8):760-4.

12. Kirby JS, Miller JJ, Adams DR, Leslie D. Health care utilization patterns and costs for patients with hidradenitis suppurativa. JAMA Dermatol. 2014;150(9):937-44.

13. Jemec GB, Kimball AB. Hidradenitis suppurativa: epidemiology and scope of the problem. J Am Acad Dermatol. 2015;73(5 Suppl 1):S4-7.

14. Tzellos T, Yang H, Mu F, Calimlim B, Signorovitch J. Impact of hidradenitis suppurativa on work loss, indirect costs and income. $\mathrm{Br} \mathrm{J}$ Dermatol. 2019;181(1):147-54.

15. Konig A, Lehmann C, Rompel R, Happle R. Cigarette smoking as a triggering factor of hidradenitis suppurativa. Dermatology. 1999;198(3):261-4.

16. Garg A, Papagermanos V, Midura M, Strunk A. Incidence of hidradenitis suppurativa among tobacco smokers: a population-based retrospective analysis in the USA. Br J Dermatol. 2018;178(3): 709-14.

17. Sabat R, Chanwangpong A, Schneider-Burrus S, Metternich D, Kokolakis G, Kurek A, et al. Increased prevalence of metabolic syndrome in patients with acne inversa. PLoS ONE. 2012;7(2):e31810.

18. Thorlacius L, Cohen AD, Gislason GH, Jemec GBE, Egeberg A. Increased suicide risk in patients with hidradenitis suppurativa. J Invest Dermatol. 2018;138(1):52-7. 
19. Machado MO, Stergiopoulos V, Maes M, Kurdyak PA, Lin PY, Wang LJ, et al. Depression and Anxiety in Adults With Hidradenitis Suppurativa: A Systematic Review and Meta-analysis. JAMA Dermatol. 2019.

20. Skroza N PI, Bernardini N, Aquila E, Balduzzi V, La Viola G, Mambrin A, Muscianese M, Tolino E, Zuber S, Potenza C, Ferrazza P. Il-17 and its role in psoriasis, hidradenitis suppurativa and acne. Internal Medicine and Care. 2017

21. Napolitano M, Megna M, Timoshchuk EA, Patruno C, Balato N, Fabbrocini G, et al. Hidradenitis suppurativa: from pathogenesis to diagnosis and treatment. Clin Cosmet Investig Dermatol. 2017;10:105-15.

22. Thomi R, Cazzaniga S, Seyed Jafari SM, Schlapbach C, Hunger RE. Association of hidradenitis suppurativa with $\mathrm{T}$ helper $1 / \mathrm{T}$ helper 17 phenotypes: a semantic map analysis. JAMA Dermatol. 2018;154(5):592-5.

23. Kridin K, Shani M, Schonmann Y, Fisher S, Shalom G, Comaneshter D, et al. Psoriasis and Hidradenitis Suppurativa: a Large-scale Population-based Study. J Am Acad Dermatol. 2018.

24. Kjaersgaard Andersen R, Saunte SK, Jemec GBE, Saunte DM. Psoriasis as a comorbidity of hidradenitis suppurativa. Int $\mathrm{J}$ Dermatol. 2020;59(2):216-20.

25. Patel M, Cohen JM, Wright NA, Merola JF, Qureshi AA, Vleugels RA. Epidemiology of concomitant psoriasis and hidradenitis suppurativa (HS): experience of a tertiary medical center. J Am Acad Dermatol. 2015;73(4):701-2.

26. Sartorius K, Emtestam L, Jemec GB, Lapins J. Objective scoring of hidradenitis suppurativa reflecting the role of tobacco smoking and obesity. Br J Dermatol. 2009;161(4):831-9.

27. Gottlieb A, Merola JF. Psoriatic arthritis for dermatologists. J Dermatol Treat. 2019:1-18.

28. von der Werth JM, Williams HC. The natural history of hidradenitis suppurativa. J Eur Acad Dermatol Venereol. 2000;14(5):389-92.

29. Schafer I, Rustenbach SJ, Radtke M, Augustin J, Glaeske G, Augustin M. Epidemiology of psoriasis in Germany-analysis of secondary health insurance data. Gesundheitswesen. 2011;73(5):308-13.

30. Matusiak L, Bieniek A, Szepietowski JC. Hidradenitis suppurativa and associated factors: still unsolved problems. J Am Acad Dermatol. 2009;61(2):362-5.
31. Cosmatos I, Matcho A, Weinstein R, Montgomery MO, Stang P. Analysis of patient claims data to determine the prevalence of hidradenitis suppurativa in the United States. J Am Acad Dermatol. 2013;68(3):412-9.

32. Jemec GB, Heidenheim M, Nielsen NH. Prevalence of hidradenitis suppurativa in Denmark. Ugeskr Laeger. 1998;160(6):847-9.

33. Vinding GR, Miller IM, Zarchi K, Ibler KS, Ellervik $\mathrm{C}$, Jemec GB. The prevalence of inverse recurrent suppuration: a population-based study of possible hidradenitis suppurativa. $\mathrm{Br} \mathrm{J}$ Dermatol. 2014;170(4):884-9.

34. Garg A, Lavian J, Lin G, Strunk A, Alloo A. Incidence of hidradenitis suppurativa in the United States: a sex- and age-adjusted population analysis. J Am Acad Dermatol. 2017;77(1):118-22.

35. Rachakonda TD, Schupp CW, Armstrong AW. Psoriasis prevalence among adults in the United States. J Am Acad Dermatol. 2014;70(3):512-6.

36. Augustin M, Reich K, Glaeske G, Schaefer I, Radtke M. Co-morbidity and age-related prevalence of psoriasis: analysis of health insurance data in Germany. Acta Derm Venereol. 2010;90(2):147-51.

37. Saunte DM, Boer J, Stratigos A, Szepietowski JC, Hamzavi I, Kim KH, et al. Diagnostic delay in hidradenitis suppurativa is a global problem. Br J Dermatol. 2015;173(6):1546-9.

38. Clerc H, Tavernier E, Giraudeau B, Bourdais-Sallot A, Samimi M, Abdo I, et al. Understanding the long diagnostic delay for hidradenitis suppurativa: a national survey among French general practitioners. Eur J Dermatol. 2018.

39. Ergun T. Hidradenitis suppurativa and the metabolic syndrome. Clin Dermatol. 2018;36(1):41-7.

40. Fimmel S, Zouboulis CC. Comorbidities of hidradenitis suppurativa (acne inversa). Dermatoendocrinology. 2010;2(1):9-16.

41. Libby P. Inflammation in atherosclerosis. Arterioscler Thromb Vasc Biol. 2012;32(9):2045-51.

42. Hansson GK. Inflammation, atherosclerosis, and coronary artery disease. $\mathrm{N}$ Engl J Med. 2005;352(16):1685-95.

43. Denny G, Anadkat MJ. The effect of smoking and age on the response to first-line therapy of hidradenitis suppurativa: an institutional retrospective cohort study. J Am Acad Dermatol. 2017;76(1):54-9. 
44. Onderdijk AJ, van der Zee HH, Esmann S, Lophaven S, Dufour DN, Jemec GB, et al. Depression in patients with hidradenitis suppurativa. J Eur Acad Dermatol Venereol. 2013;27(4):473-8.

45. Menter A. Psoriasis and psoriatic arthritis overview. Am J Manag Care. 2016;22(8 Suppl):s216-s224224.

46. Christophers E, Barker JN, Griffiths CE, Dauden E, Milligan G, Molta $C$, et al. The risk of psoriatic arthritis remains constant following initial diagnosis of psoriasis among patients seen in European dermatology clinics. J Eur Acad Dermatol Venereol. 2010;24(5):548-54.

47. De Marco G, Cattaneo A, Battafarano N, Lubrano E, Carrera CG, Marchesoni A. Not simply a matter of psoriatic arthritis: epidemiology of rheumatic diseases in psoriatic patients. Arch Dermatol Res. 2012;304(9):719-26.

48. Loget J, Saint-Martin C, Guillem P, Kanagaratnam L, Becherel PA, Nassif A, et al. Misdiagnosis of hidradenitis suppurativa continues to be a major issue. The R-ENS Verneuil study. Ann Dermatol Venereol. 2018;145(5):331-8.

49. Kirsten N, Petersen J, Hagenstrom K, Augustin M. Epidemiology of hidradenitis suppurativa in Germany-an observational cohort study based on a multisource approach. J Eur Acad Dermatol Venereol. 2020;34(1):174-9. 